\title{
Trauma Pattern in a Level I East-European Trauma Centre
}

Sorin Paun

General Surgery Department, Carol Davila University of Medicine and Pharmacy, Emergency Hospital of Bucharest, Bucharest, Romania

*Corresponding author: Sorin Paun, General Surgery Department, Carol Davila University of Medicine and Pharmacy, Emergency Hospital of Bucharest, Bucharest, Romania, E-mail: drspaun@yahoo.com

Received date: November 16, 2015; Accepted date: March 30, 2016; Published date: April 02, 2016

Copyright: (c) 2016 Paun S. This is an open-access article distributed under the terms of the Creative Commons Attribution License, which permits unrestricted use, distribution, and reproduction in any medium, provided the original author and source are credited.

\section{Commentary}

Although they are quite rare for Europe, I consider that it should be taken into discussion also injuries provided by mass casualties from wars, terrorism and collective accidents because these are representing an important issue for nowadays. The incidence of large-scale urban attacks on civilian populations has significantly increased across the globe over the past decade. Beckett et al. [1], talking about a multidisciplinary trauma team care in Kandahar, Afghanistan, concluded that The Role III Hospital was able to sustain the ability to provide multidisciplinary trauma care despite increasing combat patient volumes and translating this model to civilian institutions will offer the opportunity to enhance the triage and services rendered to busy emergency departments and mass casualty situations. The Role III Combat Hospital is similar to a Level II Trauma Centre according to American College of Surgery - Committee on Trauma guidelines and physician staff providers include: Emergency Trained Trauma Team Leaders, Anaesthesia, Orthopaedics, General Surgery, Critical Care, Internal or Family Medicine, Dental and General Radiology. Besides improvised explosive devices or severe gunshot wounds injuries, they also treated lesions due to motor vehicle collisions (202 patients) and falls (81 patients). Terrorism victims comprise the minority among trauma injured people, but this small population imposes a burden on the health care system and casualties from terrorist events are more severely injured and require more resources relative to casualties from road traffic accidents [2]

The sudden influx of patients during mass casualty events may compromise the provided quality of care and possibly impact on the medical outcomes of these patients. Using the Israeli National Trauma Registry, Peleg et al. [3] showed that mass casualty events have a significantly higher mortality comparing with non-mass casualty patients, and not manifesting substantial differences in the severity of injuries. We are also supporting the idea about the importance of a trauma registry evaluating mass casualty accidents: trauma scores can be established according to the new incoming data, prognosis values can be offered after improved statistics and time schedules for diagnosis and treatment steps can be proposed using such registers.

Another special issue is represented by trauma on motorcycle drivers: they suffer serious lesions, with mortality. It has been reported [4] that in 2007 motorcyclists were 37 times more likely to die in a motor vehicle traffic crash than passenger car occupants, and nine times more likely to be injured. Whereas car and truck fatality rates have decreased for the past 6 and 3 years, respectively, 2008 marked the eleventh consecutive year in which the annual motorcycle fatality rate increased. The rates of skull, face, spine, rib, and pelvis fractures have increased in the USA motorcycle crashes compared with historical reports [4]. The above data should be recorded by a trauma register and should be used for scientific purposes not only in USA but around the world. Preventive measures, detailed evaluations and pertinent comments on motorcycle crashes can be obtained from these data in order to improve the quality of medical acts for these special trauma problems.

\section{References}

1. Beckett A, Pelletier P, Mamczak C, Benfield R, Elster E (2012) Multidisciplinary trauma team care in Kandahar, Afghanistan: current injury patterns and care practices. Injury 43: 2072-2077.

2. Peleg K, Savitskyand B (2009) Terrorism-related injuries versus road traffic accident-related trauma: 5 years of experience in Israel. Disaster Med Public Health Prep 3: 196-200.

3. Peleg K, Rozenfeldand M, Stein M (2010) Poorer outcomes for mass casualty events victims: is it evidence based? J Trauma 69: 653-658.

4. Zettas JP, Zettasand P, Thanasophon B (1979) Injury patterns in motorcycle accidents. J Trauma 19: 833-836. 